\title{
Vessel Axis Determination Using Wave Front Propagation Analysis
}

\author{
O. Wink ${ }^{1 \star}$, W.J. Niessen ${ }^{1}$, B. Verdonck ${ }^{2}$ and M.A. Viergever ${ }^{1}$ \\ 1 Image Sciences Institute, Room E 01.334, University Medical Center Utrecht, \\ Heidelberglaan 100, 3584 CX Utrecht, The Netherlands, \\ \{onno,wiro, $\max \}$ @isi.uu.nl \\ 2 EasyVision Advanced Development, Philips Medical Systems, \\ Postbus 10000, Best, The Netherlands \\ bert.verdonck@best.ms.philips.com
}

\begin{abstract}
A method is presented that aims at finding the central vessel axis in two and three dimensional angiographic images based on a single user defined point. After the vessels in the image are enhanced using a special purpose filter, the operator is asked to point out the vessel of interest. Subsequently, a wave front propagation is started based on the response of the filter. By analyzing the evolution of the wave front, points are retrieved that are very likely to be part of the vessel of interest. These points can either be combined to form a connected structure or to retrieve the minimum cost path to the user defined point. In this paper examples of this approach are given that illustrate the performance of this method in different types of images and in situations where there is no or hardly any image evidence of the vessel at hand.
\end{abstract}

\section{Introduction}

A vast number of approaches have been proposed to facilitate the diagnosis of vessel segments requiring a different types of user interaction and empirical parameter settings. Iterative tracking or region growing procedures are frequently used, but these experience difficulties in the presence of severely stenoted regions or imaging artifacts where there is hardly any image evidence of the vessel to guide the algorithm. Figure 1a illustrates the use of an iterative approach, where the direction of the tracked axis depends merely on local information.

Several approaches have emerged that are based on the determination of a minimum cost path, which can handle these kind of situations, e.g. 1, 2, 3, 4, 5. 6. In these approaches a wave front is propagated from a source node until a target node is reached, thus forming a path of minimum cost. In this paper an approach is suggested to determine the vessel axis based on the source node alone, by analyzing the propagation of the wave front. It is assumed that the nodes that make up the vessel are visited prior to its non-vessel neighbours in the propagation of the front. These nodes are used to form the central vessel axis which can serve as input in a subsequential visualization and quantification procedure. In Section 2 the method will be described in more detail.

\footnotetext{
* This work is funded by Philips Medical Systems, Best, The Netherlands.
} 

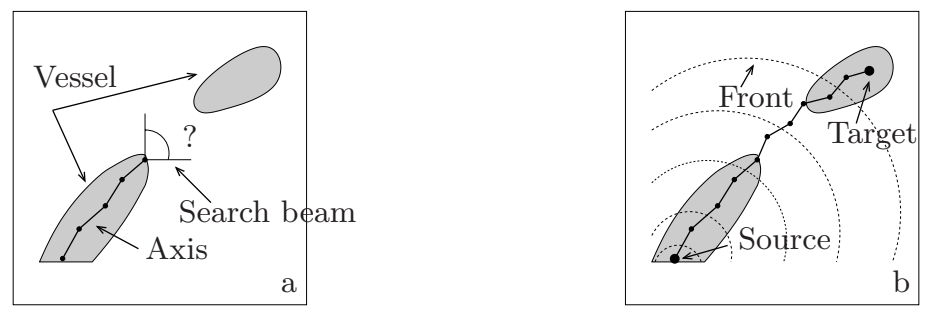

Fig. 1. Situation where an iterative line tracker, based on local information does not know how to proceed in case of a severe stenosis (a). The use of wave propagation to find a minimum cost path between a source and a target node that succeeds in determining an estimate of the vessel axis (b).

\section{Description of the Method}

In Fig. 2, different phases of the method are shown. First (section 2.1) tubular structures are enhanced in the image using a special-purpose multi-scale filter (Fig. 2b). The output of this filter is used to propagate the waves of minimum cumulative costs (section 2.2) from a user defined starting position. By analyzing the propagation of the wave front, points are selected that are very likely to be part of the vessel (section 2.3).
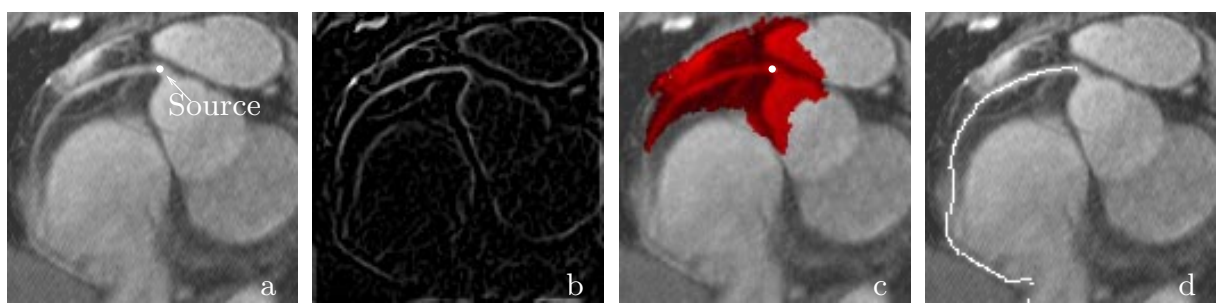

Fig. 2. A part of a slice in a MRA image containing the right coronary artery (RCA) and the user provided source point (a). The result of the eigenvalue filter (b). An instance of the wave front that is propagated (c). The "pioneer" nodes obtained $(\mathbf{d})$.

Based on these "pioneer" nodes an estimate of the central vessel axis can be obtained (section 2.6).

\subsection{Vessel Enhancement}

First, the original data is filtered with a special-purpose multi-scale filter based on the eigenvalue analysis of the Hessian matrix [7, 8, 9]. This filter is designed to highlight tubular structures in the image and is capable of coping with 
anisotropic voxels and vessels with varying width. The idea behind the eigenvalue analysis of the Hessian is to extract the principal directions in which the local second order structure of the image can be decomposed. Since this directly gives the direction of smallest curvature (along the vessel), application of several filters in multiple orientations is avoided. The latter approach is computationally more expensive and requires a discretization of the orientation space.

The second order derivative of a Gaussian used to construct the Hessian can be represented as a probe kernel that measures the contrast between the regions inside and outside the range $(-\boldsymbol{\sigma}, \boldsymbol{\sigma})$ in the direction of the derivative (see the left frame of Fig. [3). The anisotropy of the dataset is handled by adjusting the
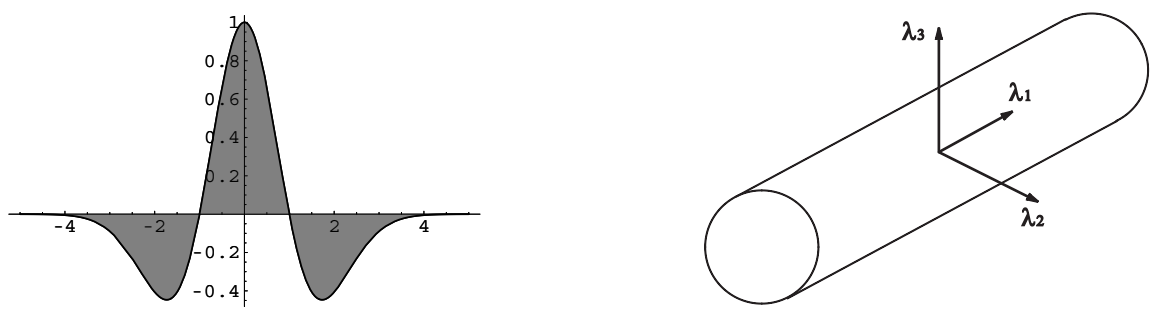

Fig. 3. Second order derivative of a Gaussian (inverse "Mexican Hat") at scale $\sigma=1$ (left) and the orientation of the three eigenvectors of the Hessian matrix when the corresponding eigenvalues are sorted in increasing magnitude (right).

parameter vector $\sigma$ in each direction. For a tubular structure, the eigenvalues, when sorted in increasing magnitude, will obey the following rules:

$$
\begin{aligned}
\left|\lambda_{1}\right| & \approx 0 \\
\left|\lambda_{1}\right| & \ll\left|\lambda_{2}\right| \\
\lambda_{2} & \approx \lambda_{3}
\end{aligned}
$$

The corresponding eigenvectors are given in the right frame of Fig. 3 The eigenvector in the direction of the vessel corresponds to the smallest eigenvalue $\lambda_{1}$, while the eigenvectors that correspond to the larger eigenvalues $\lambda_{2}$ and $\lambda_{3}$, span a plane orthogonal to the vessel. Based on these observations, Lorenz et al. 9] proposed a filter $R(\boldsymbol{\sigma})$ :

$$
\mathcal{R}(\boldsymbol{\sigma})= \begin{cases}0 & \text { if } \lambda_{2}>0 \text { or } \lambda_{3}>0 \\ \left|\lambda_{2}+\lambda_{3}\right| & \end{cases}
$$

The response of the filter is expected to be maximum at a scale that approximates the radius of the vessel. The response at different scales can be combined by taking the maximum response over a range of scales. In Fig. 2 $\mathrm{b}$ an example is given of the output of the multi-scale filter. 


\subsection{Wave Front Propagation}

There are two main approaches that can be taken to simulate the propagation of a wave front with minimum cumulative costs over a discrete grid viz. level-sets and graph-search. In the level-set approach [6] a path from one of the front nodes to the source node is determined using a backtracking procedure resulting in a sub-voxel accurate path with a true Euclidean measure. In case of the graphsearch [10], the image is treated as a grid of nodes, where every node voxel is connected to its direct neighbours. The cost of traveling from a node $n$ to its neighbour $n^{\prime}$ is given by the intensity of the voxel corresponding with $n^{\prime}$. In every iteration the neighbours of the front node having the least cumulative costs are added, thus forming all sub-paths of minimum cost. A drawback of this approach is that the resulting wave fronts are not circular and depend on the number of neighbours that are defined for an individual node. Although it is possible to trace back a path with sub-voxel accuracy from an individual front node to its source node as in the case of the level-set, a discrete version of this path can quickly be found by following the links of every predecessor of the current front node until the source node is reached. The latter is not possible in case a level-set approach is used.

The approach as suggested in this paper is not depending on the specific method used as long as for every front node, a path to its origin can be retrieved. In the remainder of the paper the graph-search method is used.

\subsection{Propagation Analysis}

From Fig. 25: it can be observed that the wave front is stretched in the direction of the vessel. In principle, the nodes in the vessels will be visited before its surrounding non-vessel nodes. This phenomenon can be used to find a path based on a single initialization, or to extrapolate a path beyond the user defined points as will be discussed in the next section. During the evolution of the front it can be monitored which nodes are the first to be expanded at a given distance from its source. The distance is defined as the length of the path from the front node to its source. These "pioneer" nodes (see Fig. 22d) are likely to be positioned in the vessel if the tortuosity of the vessel segment is not too high and the discriminative power of the filter is large. Figure 4 shows an example where the proposed method is applied on an artificially created image where a "stenosis" is introduced. From the source node as depicted in 4 a, the wave front is propagated based on the response of the filter (see Fig. 4 $\mathrm{b}$ ). If no maximum is set to the length of the minimum cost paths, every node in the image will contain the minimum costs that are needed to arrive at this node starting from the source node (see Fig. 4.). It can be appreciated that in between two adjacent vessel segments the cumulative cost are highest. As a result the pioneer nodes are first to be found inside the vessel and eventually able to overcome the loss of local information caused by the stenosis (see Fig. 4d) whereas a classical snake or connected structure will probably fail to bridge the gap. 

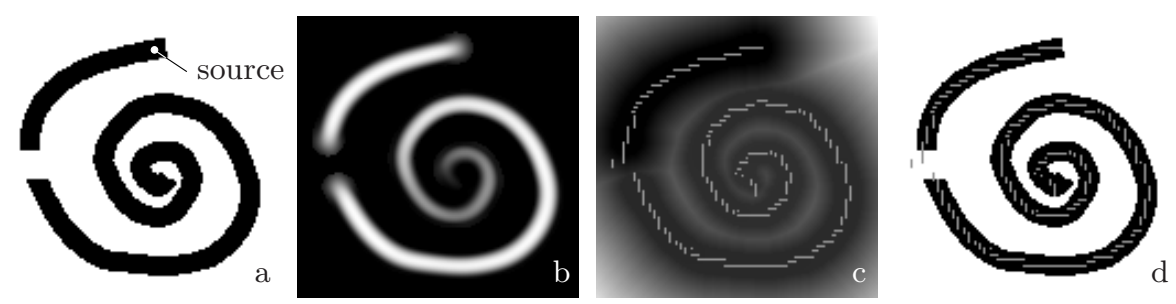

Fig. 4. Example of applying the method to an artificial image (128 x 128 pixels) where a "stenosis" is created and a source node is defined (a). The output of the eigenvalue-filter at the scale of 10 pixels(b). The image where every node contains the cost of the path back to the source node and where the "pioneer" nodes are overlayed (c). The ability of the method to overcome the stenoted region is clearly visible when the "pioneer" nodes are displayed at their corresponding position (d).

\subsection{Initialization}

If the source node is positioned in the middle of a vessel, the wave front will propagate in either direction of the vessel. As a result, the "pioneer" nodes will generally not be as close to each other as in the example of Fig. 4 $\mathrm{d}$. In this section three approaches are suggested which can be used to reduce this effect. In all three approaches two independent bounded search processes are started where the acceptance of a "pioneer" node depends on the relative position to its source node.

1. Start one bounded search process in the direction of the eigenvector corresponding to the lowest absolute eigenvector $\lambda_{1}$ which is assumed to point in the direction of the vessel (see right frame of Fig. 3). Start a second bounded search process in the opposite direction. An example is given in Fig. 5 a.

2. Start a search process for only a limited number of iterations. Find the "pioneer" node that corresponds to the largest path from the source node and use this to determine the direction of the two bounded search processes.

3. Let the user provide at least one additional node inside the vessel of interest, and use this to determine the direction of the two bounded search processes (see Fig. 5b). A traditional minimum cost path approach can be used to obtain the axis between the user provided points.

In all cases the search area can be further restricted by defining an additional angle $\alpha$ (see Fig. 5b). In Fig. 5r and Fig. 5d the second initialization method is used.

\subsection{Termination}

Since the vessels generally make up only a small portion of the scanned volume, it is very time consuming to wait until all the voxels in the image (or in the allowed 

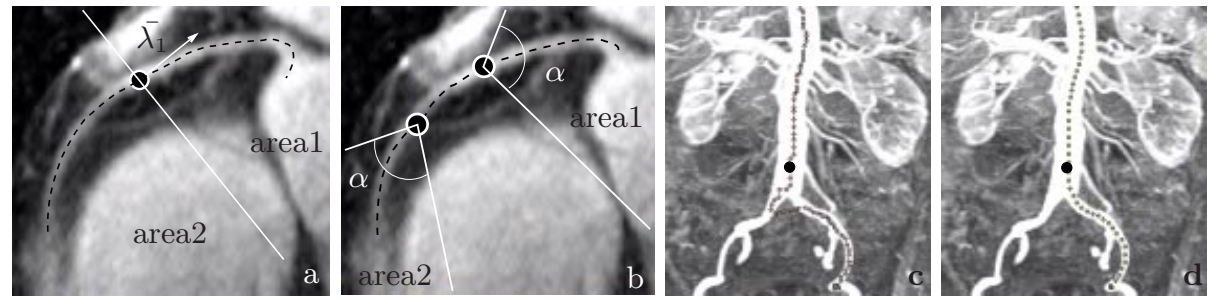

Fig. 5. Examples of bounded wave front propagation analysis. The source nodes are given by the black dots. Two areas are defined where the "pioneer" nodes of a single search process are allowed to be located. This area is based on the direction of the eigenvector $\bar{\lambda}_{1}$ in $\mathbf{a}$ and on user provided points in $\mathbf{b}$. In case of the three dimensional Contrast Enhanced MRA of the abdomen (c) the image is filtered at a wide range of scales. The "pioneer" nodes are connected and subsequently resampled to create a smooth curve which is displayed on a MIP of the original image. It is shown that the "pioneer" nodes jump from the right iliac artery to the left, a situation which can solved by computing the minimum cost paths from the last "pioneer" nodes to the source node $(\mathbf{d})$. Here the entire aorto-iliac trajectory has been outlined using a single initialization point.

areas) have been visited by the wave front. Moreover, the distances between the "pioneer" nodes will start to increase with the growing distance from its source, reducing its predictive value. It may therefore be advantageous to define one or more end-conditions which will terminate the propagation of the wave front. The most obvious end condition is to limit the maximum distance of the path to the "pioneer" nodes. Other options are e.g. to set a minimum to the required filter response, to stop the search process until the "pioneer" nodes have reached a maximum cumulative cost or the border of the image has been reached.

\section{6 "Pioneer" Nodes or Minimum Cost Path}

After the termination of the search process a list of "pioneer" nodes are retrieved. Each of these pioneer node are likely to be located in the vessel of interest. The pioneer nodes can be connected or interpolated to estimate the central vessel axis although they are not necessarily connected (see Fig. 55). Another option is to use a specific "pioneer" node as an end point and start a traditional minimum cost path approach to estimate the central vessel axis (see Fig. $5 \mathrm{j} \mathrm{d}$ ). If this "pioneer" node is very likely to be positioned in the vessel, the minimum cost path approach is the preferred method since it is guaranteed to result in a connected path which is able to cope with a stenoted region.

\section{Evaluation}

In this section the performance of the automated method using a 26-neighbourhood is compared with the performance of two observers in a task to find the 
central coronary axis from three-dimensional MRA datasets. The data is filtered with the eigenvalue filter (see Eq. 4) at a scale of $1.0 \mathrm{~mm}$. For every dataset the central coronary axis is manually determined starting from the trunk of the ascending aorta $(t)$. A total of 14 Right Coronary Arteries (RCA) are manually delineated. The process is repeated after two weeks. The manually drawn paths are averaged to form a golden standard $g$ for every dataset. This path is subsequently resampled every milimetre to form a set of sample nodes $n$ (see also the left frame of Fig. 6). For every combination between the origin $(t)$ and a sam-
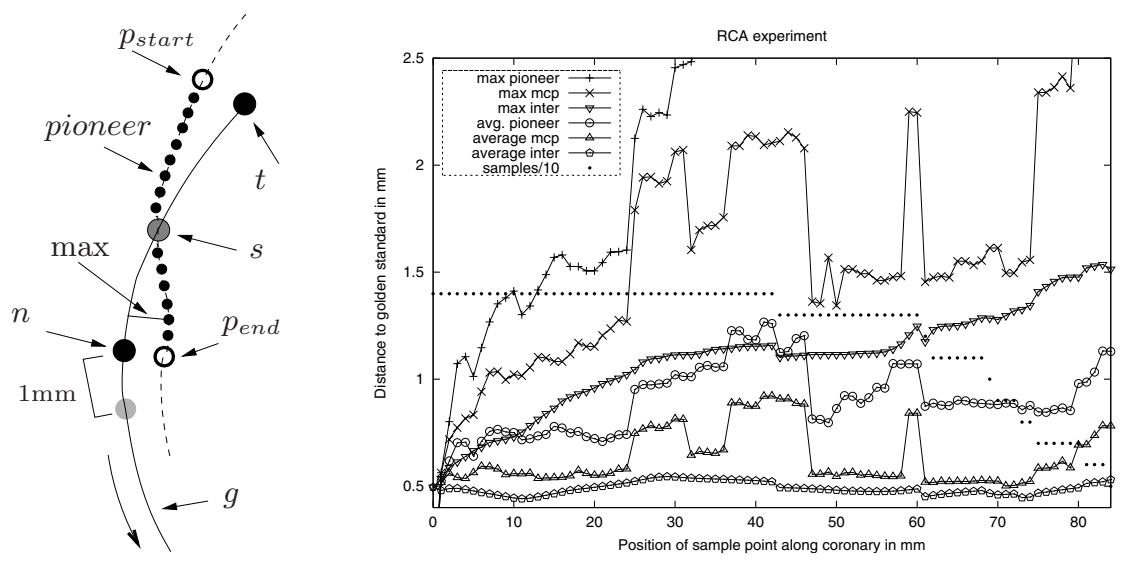

Fig. 6. Computing the maximum distance between a set of pioneer nodes originating from the source node $s$ halfway between the trunk of the ascending aorta $t$ and a sample node $n$ (left frame). The results of the evaluation (right frame).

ple node $n$, a source node $s$ is defined which is located halfway along the path between $t$ and $n$. From this source a search process is started until 10 "pioneer" nodes are found. Based on the most distant "pioneer" node the orientation of the vessel is estimated and two independent bounded search processes are started with an angle $\alpha$ of 180 degrees. The "pioneer" nodes are connected into a single axis. The subset of this axis between the "pioneer" nodes $p_{\text {start }}$ and $p_{\text {end }}$ that are closest to the nodes $t$ and $n$ make up the pioneer axis. Based on $p_{\text {start }}$, the source node $s$ and $p_{\text {end }}$, a minimum cost path $m c p$ is subsequently computed. The nodes in the axes pioneer and $m c p$ are used to compute the maximum -and average distance to the golden standard $g$. The average of these measurements over all the datasets are displayed in the right frame of Fig. 6. The differences between the two observers is given as a reference.

From the right frame of Fig. [6] it can be observed that the minimum cost path based on the last "pioneer" nodes performs consistently better than the collection of "pioneer" nodes itself. However the distances between the two observers is consistently lower than that of the automated approaches. The average distance between $m c p$ and the golden standard does not exceed $0.93 \mathrm{~mm}$, whereas the 
average in plane resolution is $0.71 \mathrm{~mm}$., and the distances between the slices is $1.5 \mathrm{~mm}$. The reason for the sudden increase of the error in the automated methods is due to the fact that in some datasets a structure close to the RCA has a higher filter output. The "pioneer" nodes are attracted to this other vessel which results in a larger error, especially if the other vessel is not running parallel to the coronary axis.

\section{Discussion and Conclusions}

A method is introduced that has the potential to obtain an estimate of the central vessel axis using only a single intialization point, by monitoring the propagation of the wave front over the voxels in the image. For the coronaries used in the evaluation, it is shown that the method performs slightly worse than a human observer, although the error does not exceed $1 \mathrm{~mm}$. The strength of the method lies on the fact that it has the potential to cope with locally disrupted data while only a limited amount of user interaction is required. Since the parameter involved in the eigenvalue-filter corresponds with an estimate of the diameter of the vessels encountered, a local estimate of the width of the vessel can be given next to the estimate of the vessel axis, providing a good starting point for subsequent visualization and quantification procedures. Although in this article the analysis of the wave front is limited to find the axis in a single vessel, the basic approach has the potential to retrieve the axes of tree-like structures as well.

\section{References}

[1] T. Y. Law and P. A. Heng, "Automatic centerline extraction for 3D virtual bronchoscopy," in Proc. Medical Image Computing and Computer-Assisted Intervention. 2000, pp. 786-795, Springer-Verlag.

[2] B. B. Avants and J. P. Williams, "An adaptive minimal path generation technique for vessel tracking in CTA/CE-MRA volume image," in Proc. Medical Image Computing and Computer-Assisted Intervention. 2000, pp. 707-715, Springer-Verlag.

[3] O. Wink, W. J. Niessen, and M. A. Viergever, "Minimum cost path determination using a simple heuristic function," in Proc. International Conference on Pattern Recognition - 4, Barcelona, 2000, pp. 1010-1013, IEEE Computer Society.

[4] A. X. Falcão, J. K. Udupa, and F. K. Miyazawa, "An ultra-fast user-steered image segmentation paradigm: Live wire on the fly," IEEE Transactions on Medical Imaging, vol. 19, no. 1, pp. 946-956, 2000.

[5] T. Deschamps, J. M. Létang, B. Verdonck, and L. D. Cohen, "Automatic construction of minimal pahts in 3D images: An application to virtual endoscopy," in Proc. Computer Assisted Radiology and Surgery,. 1999, pp. 151-155, Elsevier Publishers, Amsterdam.

[6] J. A. Sethian, Level Set Methods and Fast Marching Methods, Cambridge University Press, second edition, 1999.

[7] A. F. Frangi, W. J. Niessen, K. L. Vincken, and M. A. Viergever, "Vessel enhancement filtering," in Proc. Medical Image Computing and Computer-Assisted Intervention. 1998, pp. 130-137, Springer-Verlag. 
[8] Y. Sato et. al, "Three-dimensional multi-scale line filter for segmentation and visualization of curvilinear structures in medical images," Medical Image Analysis, vol. 2, no. 2, pp. 143-168, 1998.

[9] C. Lorenz, I. C Carlsen, T. M. Buzug, C. Fassnacht, and J. Weese, "Multi-scale line segmentation with automatic estimation of width, contrast and tangential direction in 2D and 3D medical images," in Proc. CVRMed and MRCAS, 1997, number 1205 in Lecture Notes in Computer Science, pp. 233-242.

[10] E. Dijkstra, "A note on two problems in connexion with graphs," Numerische Mathematic, vol. 1, pp. 269-271, 1959. 